\title{
RESEARCH ON THE ERRORS IN DETERMINATION OF PRECISE OPTICAL POSITIONS OF RADIO SOURCES
}

\author{
LU CHUN-LIN, LI DONG-MING \\ Purple Mountain Observatory \\ Academia Sinica \\ Nanjing, China
}

To link the optical reference frame to extragalactic radio frame, an observation program is in progress in Purple Mountain Observatory (PMO) and Shanghai Observatory. In this program, 70 extragalactic sources will be observed with the $1.56 \mathrm{~m}$ astrometric reflector in Shanghai. The secondary reference stars are determined with the twin astrograph $(D=40 \mathrm{~cm}, f=300 \mathrm{~cm})$ in PMO, and about 50 radio stars and some bright extragalactic sources will be observed with the twin astrograph.

It has been noticed that there are significant differences between optical positions of radio sources and radio stars obtained by different observers and between the optical and radio positions. Since the internal errors of these results are small, we think that these differences are mainly brought about by systematic errors. In order to obtain reliable results, research on some important systematic errors (viz. errors of reference catalogs, magnitude equation and distortion of telescope objective) has been carried out and some primary results have been obtained.

\section{Errors of reference catalogs}

To investigate the effect of errors of reference catalogs on the determination of optical positions of radio sources and radio stars, we compared AGK3RN and AGK3 - which are the most extensively used reference catalogs in the northern sky-with the FK5. In 223 fields centered at extragalactic sources selected from the IAU Commission 24 Working Group catalog (Argue et al. 1984) and radio stars selected from the High Priority Hipparcos Radio Stars (Walter 1988), the common stars are compared. There are about 9 stars in common between FK5 and AGK 3 in a $10^{\circ} \times 10^{\circ}$ field on average. Because there are too few common stars between FK5 and AGK3RN, we compared them through AGK3. In every $5^{\circ} \times 5^{\circ}$ field, the common stars between AGK3RN and AGK3 are compared. Then AGK3RN is compared with FK5 in the sense of (FK5 - AGK3) - (AGK3RN - AGK3). The results indicate that a systematic trend is not obvious in the difference between AGK3 and FK5, but it is significant in the difference between AGK3RN and FK5. On the other hand, there are a large number of fields with local difference of order of 0.11 or larger.

\section{Distortion of telescope objective}

For wide field telescopes, distortion is one of the most important aberrations which affect position determinations. We developed a new star-pair method on the basis of the classical star-pair method 
to determine distortion ( $\mathrm{Lu}$ et al. 1990). In this method, the effect of gnomonic projection on the distance between the two stars of the pair is removed using the geometry of projection. Then the coefficient of distortion can be determined with a strict reduction formula. This method eliminated the defects of the classical star-pair method. A number of star pairs on a plate can be used in the determination of distortion. With this method, we obtained distortion coefficients of the twin astrograph in PMO, which are consistent with that obtained in the solution of plate constants.

\section{Magnitude equation}

The magnitude equation is the most complicated error in photographic astrometry. It is especially important to take that equation into account in determination of optical positions of radio sources because of the large difference between magnitudes of the sources and reference stars.

We are trying out a method to determine the equation. In this method, a short exposure (1 minute) and a long exposure (30 minutes) are taken on the same plate. By analysing the relation of image diameters and the differences between the measured coordinates of two exposures, the behavior of the equation is detected, which will be used to remove the effects of the magnitude equation from the measured coordinates.

\section{References}

Argue, A.N., de Vegt, C., Elsmore, B., Fanselow, J., Harrington, R., Hemenway, P., Kuhr, H., Kumkova, I., Niell, A.E., Walter, H.G., Witzel, A.: 1984, Astron. Astrophys., 130, 191.

Lu Chun-lin, Li Dong-ming: 1990, Acta Astronomica Sinica, In press.

Walter, H.G.: 1988 , private communication.

\section{Discussion}

CoRBIN: I regret to inform you that, in my opinion, your comparison FK5 - AGK3RN is not valid for the following reasons:

1. The FK4 stars in AGK3 have positions and motions from FK4 not from the AGK3-2 program.

2. The AGK3 proper motions are not on FK4 due to the fact that FK4-FK3 was applied to AGK2, whereas AGK2 is not on FK3, but rather the system of AGK2A.

3. Thus, your in your expression (FK5 - AGK3) - (AGK3RN - AGK3) the AGK3 stars included the two sets of parentheses are not on the same system, due to the proper motions.

4. What your diagrams really show is FK4 - AGK3.

I continue to see studies that assume the proper motions of AGK3 to be on FK4, and I again caution against this.

Lu CHUN-LIN: 1. According to our analysis and the Introduction of the AGK3, the positions and proper motions of the FK4 stars in the AGK3 are not from the FK4.

2. The effect of the AGK3 proper motion system on our results is not significant. The same comparisons have been carried out at 1958.0 (the epoch of the AGK3, so there would be no effect of AGK3 proper motions) and they result in the same conclusions.

3. In the comparison of the FK5 and AGK3, the FK4 Supp stars, whose accuracy is 7 mas in position and 2 mas/y in proper motion in the FK5 system, are used. The results are consistent with FK5B.

The detailed results will soon be published elsewhere. 